\title{
Family disadvantages in early life were associated with depression in adulthood
}

\author{
Sadowski H, Ugarte B, Kolvin I, et al. Early life family disadvantages and major depression in adulthood. Br J Psychiatry 1999 \\ Feb;174:112-20.
}

\section{Question}

Are family disadvantages in early life associated with major depression in adulthood?

\section{Design}

Population based birth cohort study (Newcastle Thousand Family Study).

\section{Setting}

Newcastle upon Tyne, UK.

\section{Participants}

266 of 296 eligible participants (50\% women) from a random sample of participants in the Newcastle Thousand Family Study during their first 5 years of life. These 266 participants were assessed when they reached 33 years of age.

\section{Assessment of risk factors}

6 categories of family disadvantages were assessed: marital or family disruption, parental physical illness, poor physical care of child and home, social dependence, family overcrowding, and poor mothering (poor maternal coping skills). Multiple disadvantage was used to define those who had $\geqslant 4$ coexisting criteria of family disadvantages in their first 5 years of life.

\section{Main outcome measures}

Depressed mood or major depressive disorder (MDD) according to DSM-III-R criteria.

\section{Main results}

$10.3 \%$ of participants were depressed at follow up. Multiple disadvantages in early life were associated with depression (odds ratio [OR] 5.1, 95\% CI 2.1 to 12.0). More women than men had depres- sion (OR 2.7, CI 1.1 to 6.3). Types of disadvantages that were associated with depression were social dependence and overcrowding (OR 5.1, CI 2.1 to 12.0), poor physical care and poor mothering (OR 3.4, CI 1.4 to 7.8), and family or marital instability (OR 3.0, CI 1.3 to 6.7 ). Independent risk factors for depression were multiple disadvantages at 5 years of age $(\mathrm{p}<0.001)$ and being female $(\mathrm{p}<0.05)$ (table). Specific disadvantages that were independent risk factors for depression were family or marital instability $(\mathrm{p}<0.05)$; social dependence combined with overcrowding $(\mathrm{p}<0.05)$; and poor mothering combined with poor physical care $(\mathrm{p}<0.05)$ or female sex and poor physical care $(\mathrm{p}<0.05)$ (table).

\section{Conclusions}

The presence of multiple family disadvantages in early life was associated with depression in adulthood. Women were more likely than men to be depressed. Types of disadvantages that were associated with depression were family or marital instability, social dependence combined with overcrowding, and poor physical care combined with poor mothering.

Associations between early life disadvantages and depression

\begin{tabular}{ll}
\hline Risk factors & Adjusted OR (95\% CI)* \\
\hline Multiple disadvantages at age 5 years & $2.2(1.4$ to 3.4$)$ \\
Female sex & $1.6(1.0$ to 2.5$)$ \\
Family or marital instability & $1.7(1.1$ to 2.8$)$ \\
Social dependence and overcrowding & $2.0(1.2$ to 3.3$)$ \\
Poor physical care and poor mothering & $3.1(1.0$ to 9.1$)$ \\
Poor physical care, poor mothering, and female sex & $1.8(1.1$ to 2.9$)$
\end{tabular}

$* \mathrm{OR}=$ odds ratio, which was adjusted for sex and types of disadvantages.

Source of funding: in part, Leverhulme Emeritus Fellowship

For correspondence: Professor I Kolvin, The Tavistock Clinic, Child and Family Department, 120 Belsize Lane, London NW3 5BA, UK.

\section{Commentary}

The study by Sadowski et al provides stronger evidence for the association between multiple adverse childhood experiences and adult depression shown in previous cross sectional surveys. Identification of risk and protective factors for MDD can point to ways of intervening before the onset of disorder. At the same time, understanding the mechanisms by which family disadvantage in early life is linked with MDD remains an ongoing challenge.

Sadowski et al ask a critical question about mechanisms: why do only some respondents exposed to early life disadvantages develop a disorder? It is important to note, however, that the main outcome was 1 year prevalence of MDD; they did not measure the lifetime prevalence of disorder. Given the episodic nature of MDD, many more participants may have had $\geqslant 1$ MDD episode before 33 years of age. Understanding the pathways between predictors and outcomes over 27 years is a complex task. As Rutter and Rutter state, most risk and protective processes do not happen at one point but involve a series of chain reactions over time. ${ }^{1}$ Ideally, future longitudinal studies will collect data more frequently so that continuities and discontinuities in exposure and outcome can be examined. Furthermore, data on the family history of MDD are essential in exploring how early life disadvantages interact with genetic vulnerability in predisposing to MDD.

What can we hypothesise about early childhood interventions to prevent later depression? Clearly, developing ways to provide economic and family stability for children seems important. Indeed, the relevance of these determinants has been recognised before, but investigators and policymakers struggle with finding ways to meet the socioeconomic needs of children and families. Reduction of "poor mothering" and "poor physical care" may hold the most promise for early intervention. Olds et al have shown that prenatal and early childhood home visits by nurses can prevent parenting problems and improve child health among disadvantaged families. ${ }^{2}$ Perhaps a similar approach could be developed with the goal of interrupting the pathway between family disadvantage in childhood and adult MDD. Finally, this study shows that differences in risk factors for MDD between men and women may require developing sex specific strategies.

Harriet L MacMillan, MD, MSc, FRCP Hamilton Health Sciences Corporation Hamilton, Ontario, Canada

Jan E Fleming, MD, FRCP Toronto, Ontario, Canada

1 Rutter M, Rutter M. Developing minds: challenge and continuity across the life span. New York: and continuity across

2 Olds DL, Eckenrode J, Henderson CR Jr, et al. JAMA 1997;278:637-43. 